\title{
Carbonyl stress induces hypertension and cardio-renal vascular injury in Dahl salt-sensitive rats
}

\author{
Xianguang Chen ${ }^{1}$, Takefumi Mori ${ }^{1}$, Qi Guo ${ }^{2}$, Chunyan $\mathrm{Hu}^{1}$, Yusuke Ohsaki ${ }^{1}$, Yoshimi Yoneki ${ }^{1}$, Wanjun $\mathrm{Zhu}^{3}$, \\ Yue Jiang ${ }^{1}$, Satoshi Endo ${ }^{1}$, Keisuke Nakayama ${ }^{3}$, Susumu Ogawa ${ }^{1}$, Masaaki Nakayama ${ }^{3}$, Toshio Miyata ${ }^{4}$ and \\ Sadayoshi Ito ${ }^{1}$
}

One major precursor of carbonyl stress, methylglyoxal (MG), is elevated in the plasma of chronic kidney disease (CKD) patients, and this precursor contributes to the progression of vascular injury, hypertension and renal injury in diabetic nephropathy patients. This molecule induces salt-sensitive hypertension via a reactive oxygen species-mediated pathway. We examined the role of MG in the pathogenesis of hypertension and cardio-renal injury in Dahl salt-sensitive (Dahl S) rats, which is a rat model of CKD. Nine-week-old Dahl S rats were fed a $1 \% \mathrm{NaCl}$ diet, and $1 \% \mathrm{MG}$ was added to their drinking water for up to 12 weeks. Blood pressure and cardio-renal injuries were compared with rats treated with tap water alone. The angiotensin II receptor blocker (ARB), candesartan (10 $\left.\mathrm{mg} \mathrm{kg}^{-1} \mathrm{day}^{-1}\right)$, was administered to MG Dahl $\mathrm{S}$ rats to determine the impact of this drug on the pathogenesis of MG-induced CKD. A progressive increase in systolic blood pressure was observed $(123 \pm 1-148 \pm 5 \mathrm{~mm} \mathrm{Hg})$ after 12 weeks of MG administration. MG administration significantly increased urinary albumin excretion, glomerular sclerosis, tubular injury, myocardial collagen content and cardiac perivascular fibrosis. MG also enhanced the renal expression of $\mathrm{N} \varepsilon$ carboxyethyl-lysine (an advanced glycation end product), 8-hydroxydeoxyguanosine (a marker of oxidative stress), macrophage (ED-1) positive cells (a marker of inflammation) and nicotinamide adenine dinucleotide phosphate (NAD(P)H) oxidase activity. Candesartan treatment for 4 weeks significantly reduced these parameters. These results suggest that MG-induced hypertension and cardio-renal injury and increased inflammation and carbonyl and oxidative stress, which were partially preventable by an ARB. Hypertension Research (2013) 36, 361-367; doi:10.1038/hr.2012.204; published online 31 January 2013

Keywords: carbonyl stress; chronic kidney disease; methylglyoxal; salt sensitivity

\section{INTRODUCTION}

Methylglyoxal (MG) is a highly reactive alpha-dicarbonyl compound that is biosynthesized by the glycolysis pathway. MG modifies the lysine and arginine residues of proteins, including radical scavengers, ${ }^{1}$ and it is a potent precursor of advanced glycation end products (AGEs). ${ }^{2}$ Plasma MG levels are increased in diabetes patients and in patients with chronic kidney disease (CKD). ${ }^{3}$ Therefore, MG and its protein adducts have been implicated in the development of chronic diabetes complications, such as retinopathy and nephropathy, and uremia, which are referred to as carbonyl stress. ${ }^{2}$

We recently demonstrated that increased plasma levels of the carbonyl compound, MG, are associated with vascular and renal complications. ${ }^{4}$ MG levels correlated with increases in the intimamedia thickness of the carotid artery, systolic blood pressure (SBP), albumin-to-creatinine ratio, pulse-wave velocity and reductions in estimated glomerular filtration rate over a 5-year period. Interestingly,
MG was an independent risk factor for intima-media thickness, SBP and pulse-wave velocity after 5 years.

MG reacted with reactive oxygen species, such as hydrogen peroxide $\left(\mathrm{H}_{2} \mathrm{O}_{2}\right)$ in an in vitro study using electron spin resonance, which further exacerbated oxidative stress. ${ }^{2}$ We have demonstrated that oral MG administration to Sprague-Dawley (SD) rats induces salt sensitivity and insulin resistance concomitant with enhanced oxidative/carbonyl stress. ${ }^{5}$ These features are commonly observed in CKD. ${ }^{6}$ Therefore, we hypothesized that MG induces hypertension and vascular injury in the presence of oxidative stress, which results in cardio-renal injury in CKD.

MG was orally administered to Dahl salt-sensitive (Dahl S) rats with a low-salt diet for 4-8 weeks to investigate our hypothesis, and blood pressure and cardio-renal injury were analyzed. The Dahl S rat is an experimental model of salt-sensitive hypertension and progressive kidney disease, a disease progression that is observed in African

${ }^{1}$ Division of Nephrology, Endocrinology and Vascular Medicine, Tohoku University Graduate School of Medicine, Sendai, Japan; ${ }^{2}$ Department of Rehabilitation and Sports Medicine, Tianjin Medical University, Tianjin, China; ${ }^{3}$ Department of Blood Purification, Tohoku University Hospital, Sendai, Japan and ${ }^{4}$ United Centers for Advanced Research and Translational Medicine, Tohoku University Graduate School of Medicine, Sendai, Japan

Correspondence: Dr T Mori, Division of Nephrology, Endocrinology and Vascular Medicine, Tohoku University Graduate School of Medicine, 1-1 Seiryocho, Aoba-ku, Sendai, Miyagi 980-8574, Japan.

E-mail: tmori@med.tohoku.ac.jp

or Dr Q Guo, Department of Rehabilitation and Sports Medicine, Tianjin Medical University, 22 Qixiangtai Road, Heping District, Tianjin 300070, China.

E-mail: guoqijp@gmail.com

Received 8 May 2012; revised 6 September 2012; accepted 25 September 2012; published online 31 January 2013 
Americans. ${ }^{7}$ Renal oxidative stressors, including superoxide and $\mathrm{H}_{2} \mathrm{O}_{2}$, are enhanced in Dahl S rats even without a high-salt diet, and these stressors have a pivotal role in the impaired regulation of blood pressure. ${ }^{8,9}$ Plasma renin activity is reduced in the Dahl S model, but the local renin-angiotensin system- and angiotensin IIinduced oxidative stressors are enhanced. ${ }^{10,11}$ Therefore, the cardiorenal benefits of an angiotensin II receptor blocker (ARB), candesartan, was evaluated to determine the role of angiotensin II-mediated oxidative stress and the interaction with MG.

\section{METHODS}

\section{Animals}

Nine-week-old male Dahl S rats (Dahl S/Jr Sea, Kyudo, Yoshitomi, Japan) were housed in a temperature- and humidity-controlled room on a 12-h light-dark cycle. The rats were fed with standard $0.5 \% \mathrm{NaCl}$ rat chow (MR stock, Nihon Nosan, Yokohama, Japan). All procedures were performed in accordance with the National Institutes of Health Guide for the Care and Use of Laboratory Animals, and the animal committee of Tohoku University approved all protocols.

\section{Protocol 1: role of MG in blood pressure, renal injury and myocardial fibrosis}

This study used 9-week-old Dahl S rats to examine the effects of MG on hypertension and cardio-renal injury in a model of CKD. Dahl S rats at this age exhibit increased oxidative stress compared with age-matched salt-resistant rats. ${ }^{8}$ The rats received either $1 \% \mathrm{MG}$ in tap water (MG- $12 \mathrm{~W}$ group, $n=9$ ) or tap water alone (control group, $n=8$ ) for 12 weeks. This oral dose of MG produced plasma levels approximately 10 times higher than the control, which is equivalent to the difference between normal subjects and stage $5 \mathrm{CKD}$ patients. We have used this oral dose of MG in SD rats previously. ${ }^{5} \mathrm{~A}$ preliminary experiment revealed that MG treatment in Dahl S rats decreases food intake for 2-3 days. Therefore, the control group was pair-fed to match the salt intake. SBP was measured every week using a non-invasive tail-cuff blood pressure measurement system (Model MK-2000A, Muromachi, Tokyo, Japan). Blood pressure was monitored in a separate set of rats using a radiotelemetry transmitter (PA-C40 Data Science International, Primetec, Tokyo, Japan) that was implanted in the femoral artery as previously reported..$^{12} \mathrm{~A}$ 24-h urine sample was collected weekly in a metabolic cage, and the samples refrigerated (KN-646, Natume, Tokyo, Japan). Urinary albumin levels were measured using an automatic analyzer (Synchron-CX-3; Beckman Coulter, Fullerton, CA, USA). The rats were anesthetized at the end of the study via intraperitoneal pentobarbital $\left(60 \mathrm{mg} \mathrm{kg}^{-1}\right)$. The right kidneys were removed immediately, weighed, snap frozen in liquid nitrogen and stored at $-80^{\circ} \mathrm{C}$ for $\mathrm{NAD}(\mathrm{P}) \mathrm{H}$ oxidase assay. The left kidneys were harvested after saline perfusion through the renal artery.

\section{Protocol 2: role of the renin-angiotensin system in MG-mediated} hypertension, renal injury and myocardial fibrosis

Age-matched Dahl S rats received $1 \% \mathrm{MG}$ in tap water (MG-4W group, $n=8$ ) or MG plus $10 \mathrm{mg} \mathrm{kg}^{-1} \mathrm{day}^{-1}$ of the ARB, candesartan (Takeda Pharmaceutical, Osaka, Japan), (MG + CAND group, $n=12$ ) for 4 weeks. Control rats were pair-fed to ensure equivalent salt intake, as described in protocol 1. SBP, urinary measurements and tissue harvesting were also performed as described in protocol 1.

\section{Histological analysis}

Renal and heart tissues were fixed in either $95 \%$ ethanol or $10 \%$ neutral formalin and embedded in paraffin for histological analysis. Renal sections were stained using the Elastica-Masson method for renal injury and examined under a light microscope. The glomerular sclerosis index was determined by the grading of at least 50 glomeruli per rat, as described previously. ${ }^{13}$

Heart sections were stained with hematoxylin with $0.1 \%$ Sirius red F3BA saturated in picric acid. ${ }^{14}$ We assessed the total myocardial collagen content (that is, the percentage of Sirius red-stained collagen area to the total myocardial area) in digitalized microscopic images using Image J software. ${ }^{14}$
Perivascular adventitial fibrosis was graded as follows: normal (0), mild perivascular fibrosis (1), hypertrophy with fibrosis and some collagen within the media (2) or extensive hypertrophy and perivascular fibrosis with periadventitial mononuclear cell infiltrate (3). ${ }^{13}$

\section{Immunohistochemical staining}

The left kidney tissue was immediately fixed with 95\% ethanol (for staining osteopontin (OPN) and ED-1 antibody) or $10 \%$ formalin (for staining $\mathrm{N} \varepsilon$ carboxyethyl-lysine (CEL), 8-hydroxydeoxyguanosine (8-OHdG), and receptor for AGEs (RAGE)) and embedded in paraffin. For OPN, CEL and 8-OHdG staining, deparaffinized sections were retrieved by microwaving in $10 \mathrm{mmoll}^{-1}$ citrate buffer $(\mathrm{pH} 7.0$ ) for $5 \mathrm{~min}$. Immunohistochemical analysis was performed using antibodies against OPN (diluted 1:100, Santa Cruz Biotechnology, Santa Cruz, CA, USA, sc-10591), ED-1 (diluted 1:100, Serotec, Oxford, UK, MCA341F), CEL (diluted 1:50, TransGenic, Kobe, Japan, KH-025), 8OHdG $\left(5 \mu \mathrm{g} \mathrm{ml}^{-1}\right.$, Japan Institute for the Control of Aging, Shizuoka, Japan, MOG-020P) and RAGE (diluted 1:1000, Millipore, Carrigtwohill, Ireland, AB5484) and processed according to previously reported methods. ${ }^{5}$ Immunopositive areas were analyzed from 20 randomly captured images using Image J software. The results are expressed as the percentage of positively stained areas, except for ED-1, which is expressed as the number of immunopositive cells per slide.

\section{$\mathrm{NAD}(\mathbf{P}) \mathbf{H}$ oxidase activity}

$\mathrm{NAD}(\mathrm{P}) \mathrm{H}$ oxidase-derived superoxide anion generation was analyzed using lucigenin-enhanced chemiluminescence as described previously. ${ }^{12}$ The activity of $\mathrm{NAD}(\mathrm{P}) \mathrm{H}$ oxidase is expressed as c.p.m. per $200 \mathrm{mg}$ protein.

\section{Statistical analysis}

Data are expressed as means \pm s.e.m. The data were analyzed using the $t$-test and two-way analysis of variance for statistical comparisons of the means of all groups. Differences between the two groups were considered significant when $P<0.05$.

\section{RESULTS}

\section{MG induces hypertension and renal injury in Dahl S rats}

No significant changes in SBPs were observed in the control group throughout the 12-week experimental period (Figure 1a). However, SBP in the MG-12W group significantly increased from week 2 $(P<0.05$ vs. week 0 in the MG-12W group) to the end of the experiment, and this increase was significant compared with the control group $(P<0.05)$. Candesartan treatment significantly attenuated the MG-induced increase in SBP (Figure $1 \mathrm{~b}, P<0.05$ ).

We monitored blood pressure using radio-telemetry to confirm MG-induced hypertension and determine the diurnal variations in blood pressure. The mean blood pressure was significantly increased in the MG group compared with the control group during the active phase but not the inactive phase (Figure 1c).

Urinary albumin excretion, glomerular sclerosis index and OPN expression were significantly increased in the $\mathrm{MG}-12 \mathrm{~W}$ group compared with the control group (Figure 2 and Table $1, P<0.05$ ). We examined treatment with candesartan for 4 weeks in protocol 2 because SBP was increased from week 2 in MG-12W group. Candesartan treatment for 4 weeks decreased urinary albumin excretion, glomerular sclerosis index and OPN expression in the $\mathrm{MG}+\mathrm{CAND}$ group compared with the MG group $(P<0.05)$.

\section{Role of MG in renal inflammation and carbonyl/oxidative stress in Dahl S rats}

The number of ED-1-positive cells in the glomerulus and interstitial areas increased significantly in the MG-12W group compared with the control group (Figure 3 and Table $1, P<0.05$ ). The number of ED-1-positive cells in the glomerulus and interstitial areas decreased 

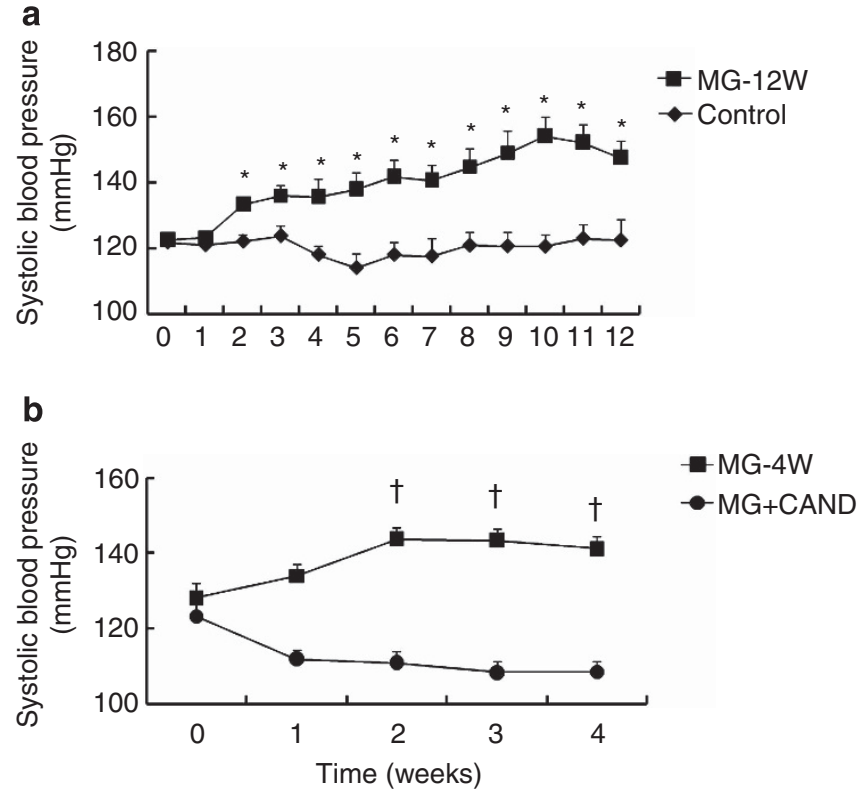

C Active phase Inactive phase

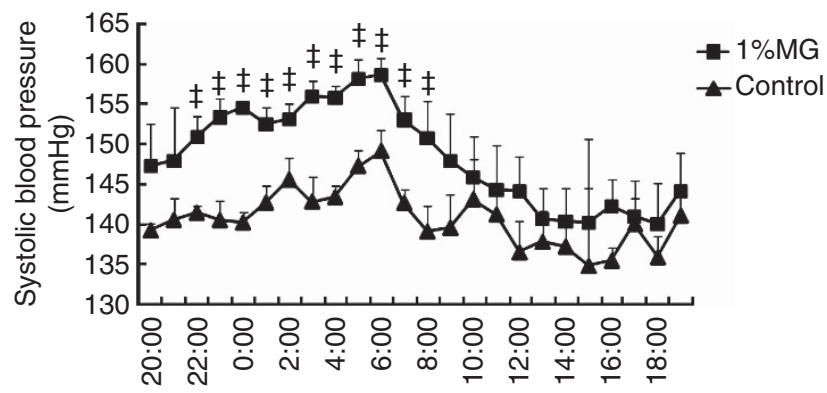

Time

Figure 1 Sequential systolic blood pressure (SBP) values measured using the tail-cuff method during the 12-week experimental period in protocol 1 (a); and during the 4-week experimental period in protocol 2 (b). Twentyfour-hour SBP values from the femoral artery measured using a radiotelemetry transmitter system (c). Protocol 1 : control $(n=8)$, treatment with tap water; MG-12W $(n=9)$, treatment with $1 \%$ methylglyoxal (MG) in drinking water for 12 weeks. Protocol 2: MG-4W $(n=8)$, treatment with $1 \%$ MG in drinking water; MG + CAND $(n=12)$, co-treatment with $M G$ and candesartan for 4 weeks. Data are presented as means \pm s.e. ${ }^{*} P<0.05$ vs. control group and data of MG- $12 \mathrm{~W}$ at 0 week; ${ }^{\dagger} P<0.05$ vs. MG + CAND group and data of MG-4W at 0 week. ${ }^{\ddagger} P<0.05$ vs. control group.

significantly in the MG + CAND group compared with the MG-4W group (Figure 3 and Table 1).

Strong CEL positivity was observed in the juxtamedullary and outer medullary regions (Figure 3). The percentage of the CELpositive area was significantly higher in the MG-12W group than the control group (Table $1, P<0.05$ ). MG + CAND treatment significantly reduced the percentage of CEL-positive regions compared with MG alone $(P<0.01)$. A significant increase in 8 -OHdG expression was observed in the kidney following MG treatment for 12 weeks (Figure 3 and Table 1). A clear decrease in 8-OHdG expression was observed in the MG + CAND compared with the MG- $4 \mathrm{~W}$ group $(P<0.05)$.

$\mathrm{NAD}(\mathrm{P}) \mathrm{H}$ oxidase activity in kidney tissue increased significantly in the MG-12W group compared with the control group (Table 1, a 24h urinary albumin excretion
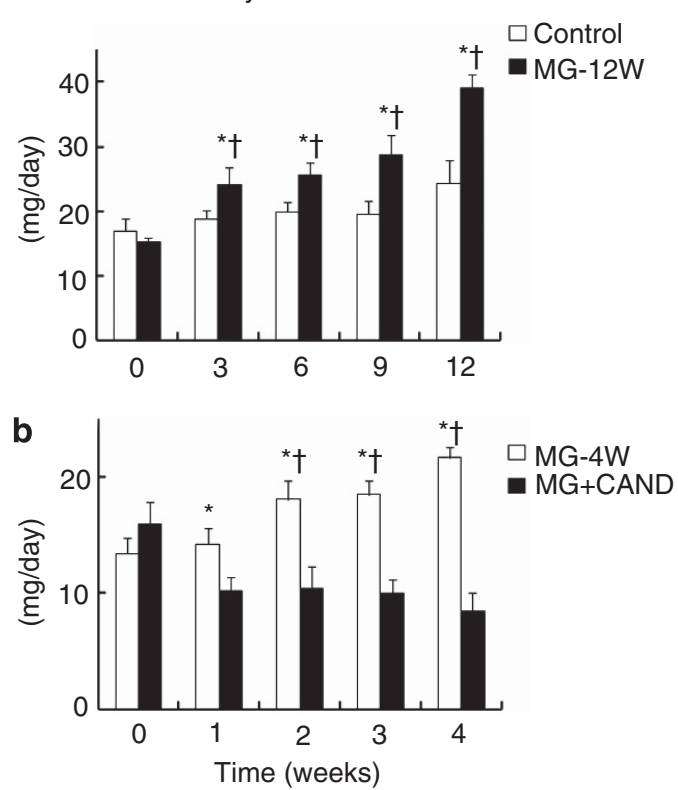

Figure 2 Twenty-four-hour urinary albumin excretion during the 12-week experimental period in protocol 1 (a), and during the 4-week experimental period in protocol 2 (b). Protocol 1: control $(n=8)$, treatment with tap water; MG-12W ( $n=9)$, treatment with $1 \%$ methylglyoxal (MG) in drinking water for 12 weeks. Protocol 2: MG-4W $(n=8)$, treatment with $1 \% \mathrm{MG}$ in drinking water; $M G+C A N D \quad(n=12)$, co-treatment with $M G$ and candesartan for 4 weeks. Data are presented as means \pm s.e. ${ }^{*} P<0.05$ vs. control or $\mathrm{MG}+\mathrm{CAND}$ group; ${ }^{\dagger} P<0.05$ vs. data of MG-12W or MG-4W group at 0 week.

$P<0.05)$. Renal NAD $(\mathrm{P}) \mathrm{H}$ oxidase activity was reduced significantly in the MG + CAND group compared with the MG group $(P<0.01)$.

RAGE expression was prominent in podocytes, the distal tubules and collecting ducts and some areas of positive CEL and 8-OHdG staining. These results suggest that the AGE-RAGE axis is partially present in MG-induced renal injury (Figure 3).

\section{MG induces cardiovascular injury in Dahl S rats}

The total myocardial collagen content and perivascular adventitial fibrosis scores were significantly higher in the MG-12W group compared with the control group, and these factors were significantly lower in the MG + CAND group compared with the MG-4W group (Table 1).

\section{DISCUSSION}

This study demonstrated that MG-induced hypertension and cardiorenal injury in a non-diabetic rat CKD model. Cardio-renal tissue damage was partially dependent on the angiotensin II type-1 receptormediated pathway, and it was associated with enhanced carbonyl and oxidative stress.

\section{Role of MG in hypertension development}

A link between MG accumulation and hypertension has been demonstrated previously in experimental animals. ${ }^{5,15,16}$ Aortic and renal MG levels increase in spontaneously hypertensive rats compared with Wistar Kyoto rats. ${ }^{15,16}$ SBP significantly increases after the oral administration of MG for 1 week in Wistar Kyoto rats. ${ }^{17}$ A diet high in fructose, which is a precursor of MG, induces hypertension and renal injury. ${ }^{18,19}$ This study confirmed that MG-induced hypertension 
Table 1 Parameters of kidney and heart tissue in protocols 1 and 2

\begin{tabular}{|c|c|c|c|c|}
\hline \multirow[b]{2}{*}{ Group } & \multicolumn{2}{|c|}{ Protocol 1} & \multicolumn{2}{|c|}{ Protocol 2} \\
\hline & Control & $M G-12 W$ & $M G-4 W$ & $M G+C A N D$ \\
\hline GSI & $1.86 \pm 0.06$ & $2.08 \pm 0.07^{*}$ & $1.69 \pm 0.05$ & $1.35 \pm 0.03^{\dagger}$ \\
\hline OPN-positive area (\% of cortex and outer medulla) & $0.50 \pm 0.10$ & $1.49 \pm 0.12^{*}$ & $1.18 \pm 0.28$ & $0.36 \pm 0.08^{\dagger}$ \\
\hline ED-1 in glomerulus (cells per glomerulus) & $0.55 \pm 0.09$ & $1.34 \pm 0.15^{*}$ & $1.26 \pm 0.09$ & $0.97 \pm 0.09^{\dagger}$ \\
\hline ED-1 in interstitial area (cells per $\times 400$ field) & $1.47 \pm 0.25$ & $3.37 \pm 0.17 *$ & $2.15 \pm 0.06$ & $1.16 \pm 0.13^{\dagger}$ \\
\hline CEL-positive area (\% of cortex and outer medulla) & $2.8 \pm 0.5$ & $17.7 \pm 5.5^{*}$ & $7.1 \pm 0.9$ & $2.8 \pm 0.3^{\dagger}$ \\
\hline 8-OhdG-positive area (\% of cortex and outer medulla) & $2.91 \pm 0.12$ & $4.10 \pm 0.22^{*}$ & $2.10 \pm 0.25$ & $1.17 \pm 0.21^{\dagger}$ \\
\hline NADPH oxidase activity (c.p.m. per 200 mg protein) & $86 \pm 3$ & $121 \pm 10^{*}$ & $143 \pm 6$ & $124 \pm 4^{\dagger}$ \\
\hline Total collagen content (\%) & $3.5 \pm 0.3$ & $6.9 \pm 0.6^{*}$ & $4.4 \pm 0.4$ & $1.4 \pm 0.1^{\dagger}$ \\
\hline Perivascular fibrosis score & $1.8 \pm 0.1$ & $2.5 \pm 0.2^{*}$ & $1.7 \pm 0.1$ & $1.2 \pm 0.2^{\dagger}$ \\
\hline
\end{tabular}

Abbreviations: CEL, Ne-carboxyethyl-lysine; ED-1, macrophage; GSI, glomerular sclerosis index; MG, methylglyoxal; NADPH, nicotinamide adenine dinucleotide phosphate; OPN, osteopontin; 8OHdG, 8-hydroxydeoxyguanosine.

Protocol 1: control $(n=8)$, tap water; MG- $12 \mathrm{~W}(n=9)$, treatment with $1 \% \mathrm{MG}$ in drinking water for 12 weeks. Protocol 2 : MG- $4 \mathrm{~W}(n=8)$, treatment with $1 \% \mathrm{MG}$ in drinking water; MG + CAND $(n=12)$, co-treatment with MG and candesartan for 4 weeks. Data are presented as means \pm s.e. ${ }^{*} P<0.01$ vs. control group; ${ }^{\dagger} P<0.05$ vs. MG-4W group.

and renal injury in Dahl S rats that were fed a normal diet and further elucidated the underlying mechanisms. Vasdev et al..$^{17}$ demonstrated that MG binds to the sulfhydryl groups of vascular $\mathrm{Ca}^{2+}$ channels, which increases cytosolic $\left[\mathrm{Ca}^{2+}\right]$ and vascular retention and leads to hypertension. We demonstrated that renal oxidative stress is another mechanism for MG-induced hypertension.

Taylor et al. ${ }^{9}$ demonstrated that renal medullary $\mathrm{H}_{2} \mathrm{O}_{2}$ concentrations are higher in Dahl S rats than salt-resistant $\left(\mathrm{SS}_{1} 3^{\mathrm{BN}}\right)$ control rats, and $\mathrm{H}_{2} \mathrm{O}_{2}$ contributes to hypertension development in Dahl $\mathrm{S}$ rats. The reduction of renal medullary oxidative stress by an $\mathrm{NAD}(\mathrm{P}) \mathrm{H}$ oxidase inhibitor, apocynin, reduces salt sensitivity in Dahl $S$ rats. ${ }^{9}$ In contrast, the renal medullary interstitial infusion of $\mathrm{H}_{2} \mathrm{O}_{2}$ in $\mathrm{SS}_{13}{ }^{\mathrm{BN}}$ rats induces hypertension. ${ }^{20}$ These results indicate that enhanced renal oxidative stress underlies the hypertension development. However, an $8 \%$ salt diet does not produce hypertension in SD rats despite enhanced renal oxidative stress, ${ }^{12}$ and $1 \% \mathrm{MG}$ in drinking water does not produce hypertension in $\mathrm{SD}$ rats. ${ }^{5}$ A high-salt diet in SD rats treated with the same dose of MG surprisingly produces hypertension and enhances renal oxidative stress, which suggests that MG induces salt sensitivity in these rats. ${ }^{5}$ These previous results and this study suggest that MG causes hypertension in rats with enhanced renal oxidative stress. This hypothesis explains an oral dose of MG that does not induce hypertension in SD rats produces hypertension in Dahl $\mathrm{S}$ rats, which exhibit high renal $\mathrm{H}_{2} \mathrm{O}_{2}$ levels even in the absence of a highsalt diet. $^{20}$

\section{MG-induced oxidative stress and inflammation}

Several mechanisms may be involved in MG-induced renal oxidative stress. First, this study demonstrated that MG-enhanced renal $\mathrm{NAD}(\mathrm{P}) \mathrm{H}$ oxidase activity. This result is consistent with a previous study that demonstrated an MG-induced increase in $\mathrm{NAD}(\mathrm{P}) \mathrm{H}$ oxidase expression. ${ }^{21}$ Second, $\mathrm{MG}$ stimulates mitochondrial oxidative stress. ${ }^{22}$ Mitochondria and $\mathrm{NAD}(\mathrm{P}) \mathrm{H}$ oxidase are two primary sources of superoxide production in the kidney, in particular the renal medulla. ${ }^{23}$ Therefore, these pathways may also be involved in MG-induced enhancement of renal oxidative stress. Third, the MG-induced impairment of glutathione peroxidase (GSH$\mathrm{Px})$ and glutathione reductase (GSSG-Red $)^{15}$ would also increase oxidative stress because GSSG-Red has an important antioxidant defense role by reducing GSSG to GSH. In contrast, GSH-Px scavenges peroxides, which can be converted to very reactive free radicals using GSH as the electron donor. ${ }^{24}$ Fourth, RAGE contributes to the pathogenesis of renal injury, and $\mathrm{NAD}(\mathrm{P}) \mathrm{H}$ oxidase is enhanced by the RAGE-mediated pathway. ${ }^{25}$ These results are consistent with this study of enhanced renal $\mathrm{NAD}(\mathrm{P}) \mathrm{H}$ oxidase in MG-treated Dahl S rats. However, further investigation is required to determine RAGE pathway-mediated alterations in blood pressure. Fifth, we recently demonstrated that an increase in renal perfusion pressure induces renal oxidative stress. ${ }^{26}$ Therefore, we cannot exclude the possibility that enhanced oxidative stress was partially due to MG-mediated hypertension. Finally, MG reacts with $\mathrm{H}_{2} \mathrm{O}_{2}$ and induces further reactive oxygen species in vitro. ${ }^{27}$ Therefore, the high renal $\mathrm{H}_{2} \mathrm{O}_{2}$ levels in Dahl $\mathrm{S}$ rats may have interacted with $\mathrm{MG}$ to further enhance renal oxidative stress and elevate blood pressure.

The precise mechanism was not determined in this study, but the results suggest that $\mathrm{MG}$-enhanced renal inflammation. ${ }^{28}$ Oxidative stress is an initiator of inflammation and vice versa, and MG may have induced an inflammatory reaction via the oxidative stress pathway. We previously demonstrated that reductions in renal perfusion pressure reduces the number of renal ED-1-positive cells in Dahl S rats. ${ }^{29}$ Therefore, MG-induced hypertension was partially responsible for the induction of the inflammatory changes in this study.

\section{MG induces hypertension and renal injury through} the renin-angiotensin system

Angiotensin II produces renal oxidative stress via $\mathrm{NAD}(\mathrm{P}) \mathrm{H}$ oxidase. ${ }^{30}$ Angiotensin II-induced oxidative stress is enhanced in Dahl S rats compared with control salt-resistant $\mathrm{SS}_{3} 3^{\mathrm{BN}}$ rats, and it is responsible for blood pressure control. Fructose, which is a precursor of carbonyl stress, induces hypertension and insulin resistance, which is inhibited by losartan treatment. ${ }^{18}$ These results suggest that the renin-angiotensin system is responsible for carbonyl stress-induced hypertension and insulin resistance. ${ }^{5}$ The ARB, candesartan, reduced MG-induced renal injury and AGE accumulation in this study. This result is consistent with previous reports that candesartan reduces renal AGE accumulation in diabetic mice. ${ }^{31}$ The superoxide response to angiotensin II is enhanced in tissues that are isolated from the renal outer medulla of Dahl S rats compared with SS-13 ${ }^{\mathrm{BN}}$ rats. ${ }^{32}$ These studies suggest that oxidative 
a

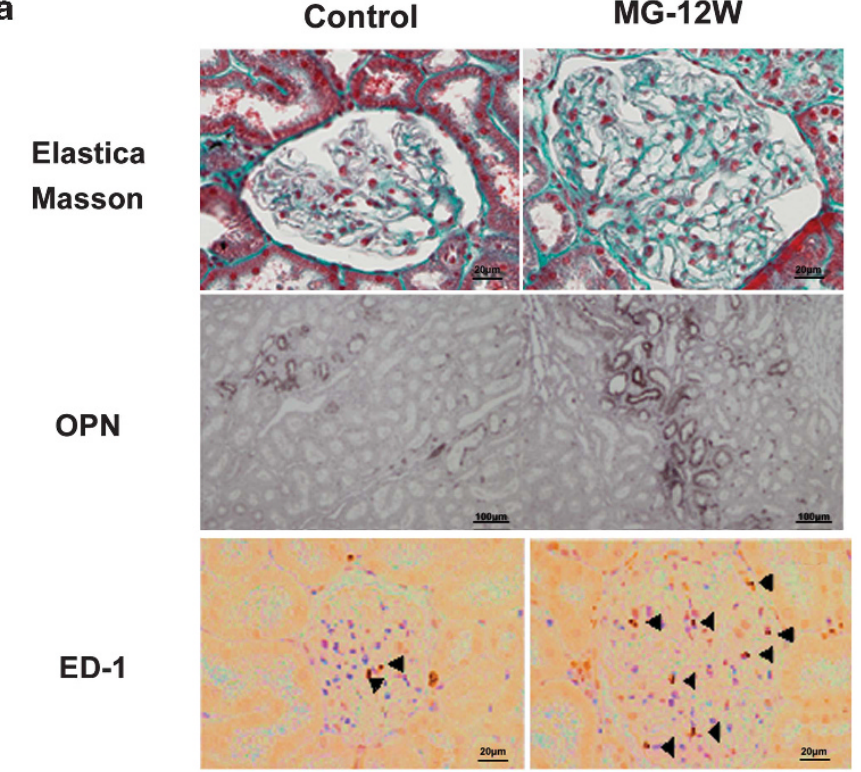

CEL

8-OHdG
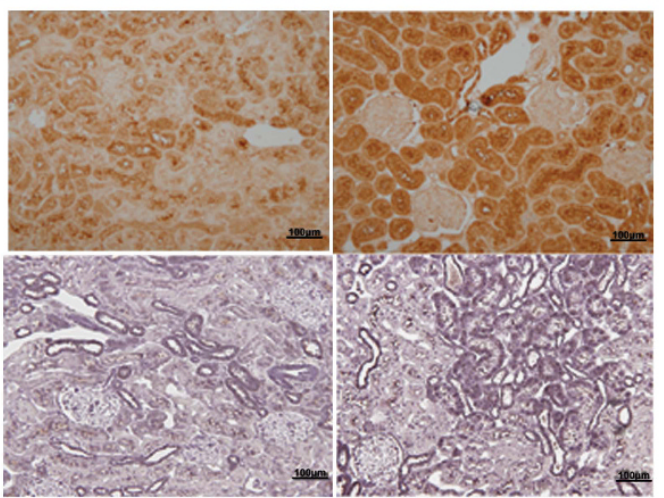

RAGE
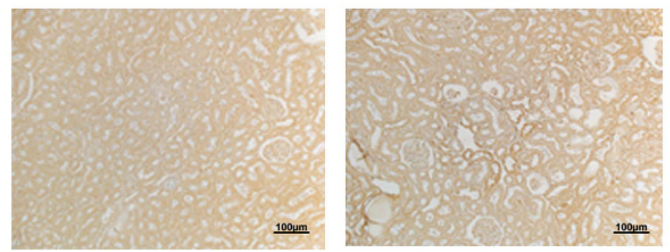

b

Collagen deposition
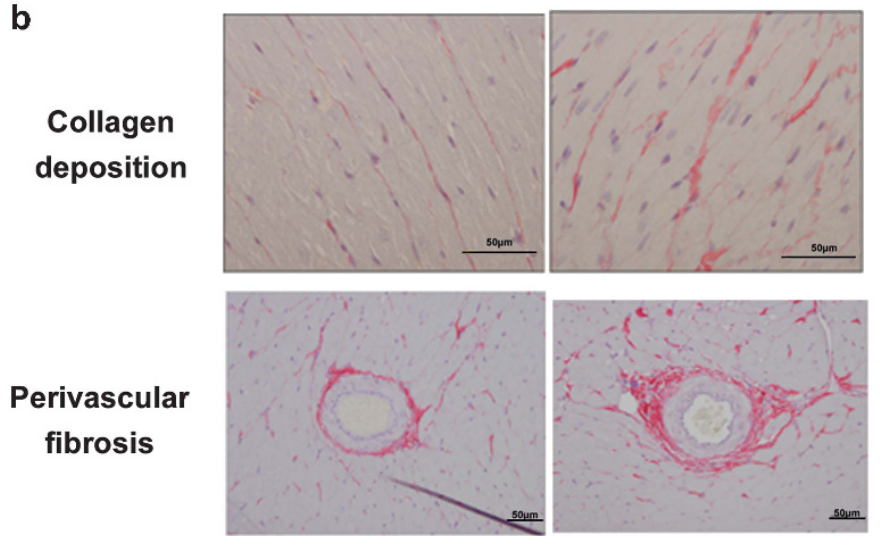
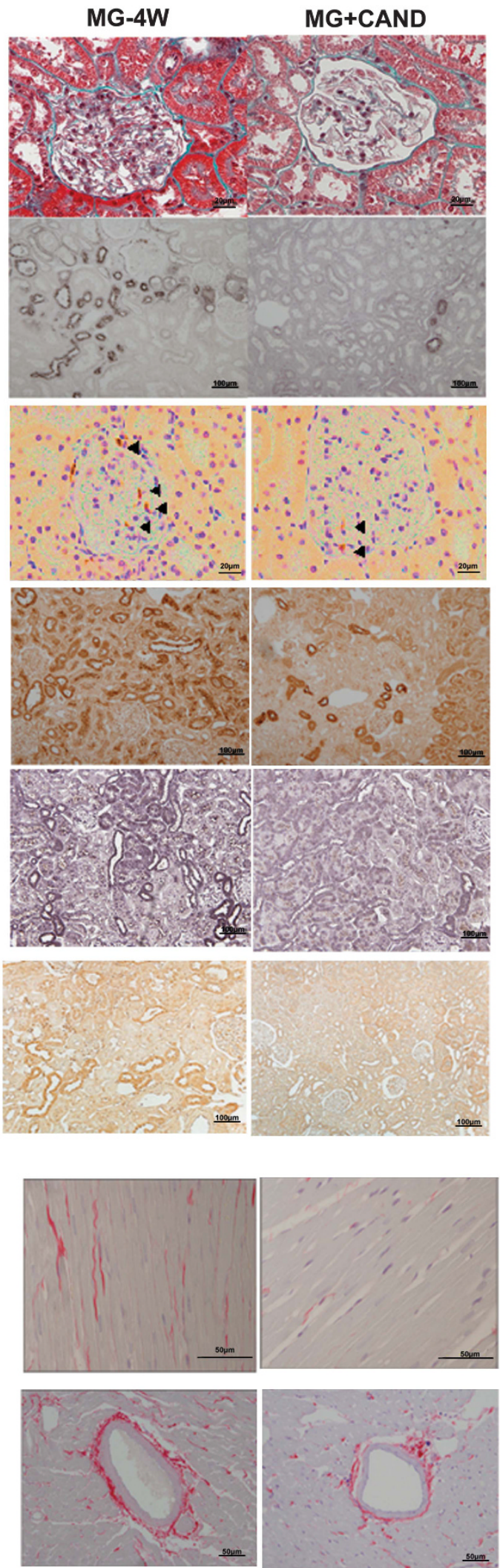

Figure $\mathbf{3}$ Images of glomerular sclerosis stained with the Elastica-Masson method and immunostained with Ne-carboxyethyl-lysine (CEL), 8hydroxydeoxyguanosine (8-OhdG), osteopontin (OPN), ED-1 antibodies (black arrowheads) and receptor for advanced glycation end products (RAGEs) in the kidney (a), and collagen deposition in the myocardial interstitial space and cardiac perivascular fibrosis in the heart (b), in protocol 1 (control and MG-12W groups treated for 12 weeks), and protocol 2 (MG-4W, MG + CAND, and CAND groups treated for 4 weeks). 
stress contributes to the salt sensitivity of Dahl $S$ rats, which is partially mediated through the renin-angiotensin system. The activation of angiotensin II type-1 receptors stimulates the generation of superoxide by $\mathrm{NAD}(\mathrm{P}) \mathrm{H}$ oxidase. ${ }^{30}$ In addition, $\mathrm{MG}$ increases angiotensinogen. ${ }^{23}$ However, a 4 -week candesartan treatment did not significantly alter plasma MG levels, despite the reduction in AGE accumulation (data not shown). The results of this study suggest that MG-enhanced $\mathrm{NAD}(\mathrm{P}) \mathrm{H}$ oxidase activity via an angiotensin IImediated pathway, which enhanced renal oxidative stress and induced hypertension and renal injury.

\section{Role of MG in myocardial fibrosis}

MG also induced myocardial and perivascular fibrosis in Dahl S rats. Candesartan treatment improved the MG-induced myocardial and perivascular fibrosis that was associated with the decrease in blood pressure. These results suggest that MG-induced hypertension produced myocardial and perivascular fibrosis via a RAS-mediated pathway. However, further investigation is required to determine whether the fibrosis was mediated via the blood pressure or the direct action of MG. This study also provides evidence that MG-induced carbonyl stress in CKD contributed to cardio-renal injury.

This study suffers one notable limitation. We assessed the maximum blood pressure response and used the highest possible concentration of MG because of the limited sensitivity in the detection of hypertension. We selected 1\% MG because this dose is the highest dosage that has been used in previous rat studies. ${ }^{5}$ However, 4 weeks of $1 \%$ MG treatment in the drinking water increased plasma MG levels in Dahl S rats <10-fold compared with control rats in this study $\left(3367 \pm 307 \mathrm{nmoll}^{-1}, n=8\right.$ vs. $253 \pm 5 \mathrm{nmoll}^{-1}, \quad n=6$ ). Plasma MG levels were assayed using derivatization with o-phenylenediamine and electrospray ionization liquid chromatography-mass spectrometry of the resulting quinoxalines, as reported previously. ${ }^{4}$ The increase in rat serum MG levels was equivalent to the increase in patients with stage $5 \mathrm{CKD}$ compared with healthy controls. ${ }^{3}$ Blood pressure responses to this dose of MG in this study were within physiological limits because the response was completely inhibited with candesartan. We could not determine whether the effects of MG on hypertension were dose dependent because of the technical limitations of measurements and the control of plasma and/or tissue concentration of MG.

The kidney injury and oxidative stress levels in the Dahl S rat model of CKD are affected by a high-salt diet and aging. ${ }^{8,33}$ Our results showed that the absolute value of 24 -h urinary albumin excretion was higher (but no significantly) in 21-week-old control rats in protocol 1 than 13-week-old MG-4W rats in protocol 2 (Figure 2), which is consistent with previous studies. Therefore, the reasons that the glomerular sclerosis index and 8-OHdG-positive areas in the controls in protocol 1 were greater than the MG- $4 \mathrm{~W}$ in protocol 2 may include the older age of the rats in protocol 1 than protocol 2, despite MG administration.

\section{PERSPECTIVES}

Carbonyl stress contributes to the pathogenesis of CKD and cardiovascular disease, and it is concomitant with salt-sensitive hypertension and insulin resistance. Enhanced oxidative stress and inflammatory markers are often observed in CKD patients, and $\mathrm{ARB}$ can reduce these factors, which is consistent with this study. Therefore, carbonyl compounds, such as MG, may be a target for the treatment of CKD and cardiovascular disease and a marker of disease pathogenesis.

\section{ACKNOWLEDGEMENTS}

This study was supported by Grants-in-Aid for Scientific Research from the Ministry of Education, Culture, Sports, Science and Technology of Japan (nos. 17590181 and 20590970). In addition, we thank Kiyomi Kisu for her expert technical assistance.

1 Lo TW, Westwood ME, McLellan AC, Selwood T, Thornalley PJ. Binding and modification of proteins by methylglyoxal under physiological conditions. A kinetic and mechanistic study with $\mathrm{N}$ alpha-acetylarginine, $\mathrm{N}$ alpha-acetylcysteine, and $\mathrm{N}$ alphaacetyllysine, and bovine serum albumin. J Biol Chem 1994; 269: 32299-32305.

2 Bourajjaj M, Stehouwer CD, van Hinsbergh VW, Schalkwijk CG. Role of methylglyoxal adducts in the development of vascular complications in diabetes mellitus. Biochem Soc Trans 2003; 31: 1400-1402.

3 Nakayama K, Nakayama M, Iwabuchi M, Terawaki H, Sato T, Kohno M, Ito S. Plasma alpha-oxoaldehyde levels in diabetic and nondiabetic chronic kidney disease patients. Am J Nephrol 2008; 28: 871-878.

4 Ogawa S, Nakayama K, Nakayama M, Mori T, Matsushima M, Okamura M, Senda M Nako K, Miyata T, Ito S. Methylglyoxal is a predictor in type 2 diabetic patients of intimamedia thickening and elevation of blood pressure. Hypertension 2010; 56: 471-476.

5 Guo Q, Mori T, Jiang Y, Hu C, Osaki Y, Yoneki Y, Sun Y, Hosoya T, Kawamata A, Ogawa $\mathrm{S}$, Nakayama M, Miyata T, Ito S. Methylglyoxal contributes to the development of insulin resistance and salt sensitivity in Sprague-Dawley rats. J Hypertens 2009; 27: 1664-1671.

6 Sarafidis PA, Grekas DM. Insulin resistance and oxidant stress: an interrelation with deleterious renal consequences? J Cardiometab Syndr 2007; 2: 139-142.

7 Johnson RJ, Gordon KL, Giachelli C, Kurth T, Skelton MM, Cowley AW Jr. Tubulointer stitial injury and loss of nitric oxide synthases parallel the development of hypertension in the Dahl-SS rat. J Hypertens 2000; 18: 1497-1505.

8 Meng S, Roberts LJ II, Cason GW, Curry TS, Manning RD Jr. Superoxide dismutase and oxidative stress in Dahl salt-sensitive and -resistant rats. Am J Physiol Regul Integr Comp Physiol 2002; 283: R732-R738.

9 Taylor NE, Glocka P, Liang M, Cowley AW Jr. NADPH oxidase in the renal medulla causes oxidative stress and contributes to salt-sensitive hypertension in Dahl $\mathrm{S}$ rats. Hypertension 2006; 47: 692-698.

10 Chandramohan G, Bai Y, Norris K, Rodriguez-Iturbe B, Vaziri ND. Effects of dietary salt on intrarenal angiotensin system, NAD(P)H oxidase, COX-2, MCP-1 and PAI-1 expressions and NF-kappaB activity in salt-sensitive and -resistant rat kidneys. Am J Nephrol 2008; 28: 158-167.

11 Kobori H, Nishiyama A, Abe Y, Navar LG. Enhancement of intrarenal angiotensinogen in Dahl salt-sensitive rats on high salt diet. Hypertension 2003; 41: 592-597.

12 Endo S, Mori T, Yoneki Y, Nakamichi T, Hosoya T, Ogawa S, Tokudome G, Hosoya T, Miyata T, Ito S. Blockade of angiotensin II type-1 receptor increases salt sensitivity in Sprague-Dawley rats. Hypertens Res 2009; 32: 513-519.

13 Lea WB, Kwak ES, Luther JM, Fowler SM, Wang Z, Ma J, Fogo AB, Brown NJ. Aldosterone antagonism or synthase inhibition reduces end-organ damage induced by treatment with angiotensin and high salt. Kidney Int 2009; 75: 936-944.

14 Mito S, Ozono R, Oshima T, Yano Y, Watari Y, Yamamoto Y, Brydun A, Igarashi K, Yoshizumi M. Myocardial protection against pressure overload in mice lacking Bach1, a transcriptional repressor of heme oxygenase-1. Hypertension 2008; 51: 1570-1577.

$15 \mathrm{Wu} \mathrm{L}$, Juurlink $\mathrm{BH}$. Increased methylglyoxal and oxidative stress in hypertensive rat vascular smooth muscle cells. Hypertension 2002; 39: 809-814.

16 Wang X, Desai K, Clausen JT, Wu L. Increased methylglyoxal and advanced glycation end products in kidney from spontaneously hypertensive rats. Kidney Int 2004; 66: 2315-2321.

17 Vasdev S, Ford CA, Longerich L, Parai S, Gadag V, Wadhawan S. Aldehyde induced hypertension in rats: prevention by $\mathrm{N}$-acetyl cysteine. Artery 1998; 23: 10-36.

18 Guo Q, Mori T, Jiang Y, Hu C, Ohsaki Y, Yoneki Y, Nakamichi T, Ogawa S, Sato H, Ito S. Losartan modulates muscular capillary density and reverses thiazide diuretic-exacerbated insulin resistance in fructose-fed rats. Hypertens Res 2012; 35: 48-54.

19 Sanchez-Lozada LG, Tapia E, Jimenez A, Bautista P, Cristobal M, Nepomuceno T, Soto V, Avila-Casado C, Nakagawa T, Johnson RJ, Herrera-Acosta J, Franco M. Fructoseinduced metabolic syndrome is associated with glomerular hypertension and renal microvascular damage in rats. Am J Physiol Renal Physiol 2007; 292: F423-F429.

20 Taylor NE, Cowley AW Jr. Effect of renal medullary H2O2 on salt-induced hypertension and renal injury. Am J Physiol Regul Integr Comp Physiol 2005; 289: R1573-R1579.

21 Bhattacharyya N, Pal A, Patra S, Haldar AK, Roy S, Ray M. Activation of macrophages and lymphocytes by methylglyoxal against tumor cells in the host. Int Immunophar macol 2008; 8: 1503-1512.

22 Rabbani N, Thornalley PJ. Dicarbonyls linked to damage in the powerhouse: glycation of mitochondrial proteins and oxidative stress. Biochem Soc Trans 2008; 36: 10451050.

23 Schupp N, Schinzel R, Heidland A, Stopper H. Genotoxicity of advanced glycation end products: involvement of oxidative stress and of angiotensin II type 1 receptors. Ann NY Acad Sci 2005; 1043: 685-695.

24 Juurlink $\mathrm{BH}$. Management of oxidative stress in the CNS: the many roles of glutathione. Neurotox Res 1999; 1: 119-140.

25 Thallas-Bonke V, Thorpe SR, Coughlan MT, Fukami K, Yap FY, Sourris KC, Penfold SA Bach LA, Cooper ME, Forbes JM. Inhibition of NADPH oxidase prevents advanced glycation end product-mediated damage in diabetic nephropathy through a protein kinase C-alpha-dependent pathway. Diabetes 2008; 57: 460-469. 
26 Jin C, Hu C, Polichnowski A, Mori T, Skelton M, Ito S, Cowley AW Jr. Effects of renal perfusion pressure on renal medullary hydrogen peroxide and nitric oxide production. Hypertension 2009; 53: 1048-1053.

27 Nakayama M, Saito K, Sato E, Nakayama K, Terawaki H, Ito S, Kohno M. Radical generation by the non-enzymatic reaction of methylglyoxal and hydrogen peroxide. Redox Rep 2007; 12: 125-133.

28 Uribarri J, Cai W, Peppa M, Goodman S, Ferrucci L, Striker G, Vlassara H. Circulating glycotoxins and dietary advanced glycation endproducts: two links to inflammatory response, oxidative stress, and aging. J Gerontol A Biol Sci Med Sci 2007; 62: 427-433.

29 Mori T, Polichnowski A, Glocka P, Kaldunski M, Ohsaki Y, Liang M, Cowley AW Jr. High perfusion pressure accelerates renal injury in salt-sensitive hypertension. J Am Soc Nephrol 2008; 19: 1472-1482.

30 Landmesser U, Cai H, Dikalov S, McCann L, Hwang J, Jo H, Holland SM, Harrison DG. Role of $\mathrm{p} 47$ (phox) in vascular oxidative stress and hypertension caused by angiotensin II. Hypertension 2002; 40: 511-515.
31 Fan Q, Liao J, Kobayashi M, Yamashita M, Gu L, Gohda T, Suzuki Y, Wang LN, Horikoshi S, Tomino Y. Candesartan reduced advanced glycation end-products accumulation and diminished nitro-oxidative stress in type 2 diabetic $\mathrm{KK} / \mathrm{Ta}$ mice. Nephrol Dial Transplant 2004; 19: 3012-3020.

32 Mori T, Cowley AW Jr. Role of pressure in angiotensin II-induced renal injury: chronic servo-control of renal perfusion pressure in rats. Hypertension 2004; 43: 752-759.

33 Meng S, Cason GW, Gannon AW, Racusen LC, Manning RD Jr. Oxidative stress in Dahl salt-sensitive hypertension. Hypertension 2003; 41: 1346-1352.

(c) (i) (2) (2) This work is licensed under a Creative Commons Attribution-NonCommercial-ShareAlike 3.0 Unported License. To view a copy of this license, visit http:// creativecommons.org/licenses/by-nc-sa/3.0/ 